\title{
A GENERALIZATION OF THE STRONGLY CESÁRO IDEAL CONVERGENCE THROUGH DOUBLE SEQUENCE SPACES
}

\author{
Carlos Granados \\ Universidad del Atlántico \\ Barranquilla, COLOMBIA
}

\begin{abstract}
In this paper, some algebraic properties of Cesáro double ideal convergent sequence spaces are defined and proved. Those spaces are defined as $C_{n m}^{I}$ and $C_{0 n m}^{I}$. Furthermore, this paper shows some inclusion relations on these spaces which are established and proved.
\end{abstract}

AMS Subject Classification: 40C05, 46A45, 46E30, 46E40, 46B20 Key Words: $C_{n m}^{I}$ space; $C_{0 n m}^{I}$ space; double ideal sequence

\section{Introduction and preliminaries}

The notion of strong Cesáro convergence was initially defined by [2], this notion was defined as: A sequence $\left(x_{n}\right)$ on a normed space $(X,\|\cdot\|)$ is called strongly Cesáro convergence to $L$ if

$\lim _{n \rightarrow \infty} 1 / k \sum_{n=1}^{k}\left\|x_{n}-L\right\|=0$. In $[4,9]$, the authors have extended this notion in several fields. Fast [10] in 1951 introduced the concept of statistical convergence, at the same time Steinhaus [11] in 1951 by his own way defined the notion of ordinary and asymptotic convergences. After that, Fridy [12, 13] studied the statistical convergence and he created a relation which linked this notion with the notion of summability theory. On the other hand, Kostyrko et al. [14] in 2000 showed the concept of ideal convergence which was a generalization of statistical convergence. Some years after, Salat et al. [5] studied

Received: December 2, 2020

(C) 2021 Academic Publications 
some properties of $I$-convergence, and then it has been studied by many mathematicians. Recently, in 2020, Faisal [1] defined the concept of strongly Cesáso ideal convergent and proved some properties.

Now, we show some definitions and previous results which are useful for the developing of this paper.

Definition 1. ([3]) Let $2 \omega=\left\{x=\left(x_{n m}\right): x_{n m} \in R\right.$ or $\left.C\right\}$ of all real and complex double sequences, where $R$ and $C$ denote the real number set and complex number set, respectively.

Definition 2. ([3]) A double sequence $x=\left(X_{n m}\right) \in 2 \omega$ is said to be double $I$-convergent to a number $L$ if for every $\epsilon>0$, we have

$$
\left\{(m, n) \in N \times N:\left|x_{n m}-L\right| \geq \epsilon\right\} \in I .
$$

In this case, we write $I$ - $\lim x_{n m}=L$.

Definition 3. ([14]) Let $X$ be a non-empty set. Then a family of sets $I \subseteq 2^{X}$ is said to be an ideal in $X$ if the following statements hold:

1. $\emptyset \in I$.

2. If $A \subset B$ and $B \in I$, then $A \in I$.

3. If $A, B \in I$, then $A \cup B \in I$.

Moreover, and ideal $I$ is called admissible if $\{\{x\}: x \in X\} \subseteq I$.

Definition 4. ([3]) A non-empty set $F \in 2^{X}$ is known as a filter in $N$ if satisfies the following statements:

1. $\emptyset \in F$.

2. $A, B \in F$, then $A \cap B \in F$.

3. $A \in F$ with $A \subseteq B$, then $B \in F$.

Remark 5. For every ideal $I$, there is a filter $F(I)$ (associated with $I$ ) defined as $F(I)=\left\{P \subseteq N \times N: P^{c} \in I\right.$ where $\left.P^{c}=N \times N-P\right\}$.

Lemma 6. (See [5]) Let $W \in F(I)$ and $M \subseteq N$. If $M \notin I$, then $M \cap W \notin I$. 
Lemma 7. (See [5]) Let $I \subseteq 2^{N}$ and $M \subseteq N$. If $M \notin I$, then $M \cap W \notin I$ and $W \in F(I)$.

Definition 8. A double sequence space of $X$ is said to be solid if $\left(\gamma_{n m} x_{n m}\right) \in$ $X$ whenever $\left(x_{n m}\right) \in X$ and $\left(\gamma_{n m}\right)$ is a sequence of scalars with $\left|\gamma_{n m} x_{n m}\right| \leq 1$, for all $(n, m) \in N \times N$.

Definition 9. A double sequence of $X$ is said to be a sequence algebra if for every $\left(x_{n m}\right),\left(y_{n m}\right) \in X,\left(x_{n m}, y_{n m}\right) \in X$.

\section{Results}

We first introduce the notion of $C_{n m}^{I}$ which denotes the space of all Cesáro double $I$-convergent sequences. Besides, we also introduce the notion of $C_{0 \mathrm{~nm}}^{I}$ which denotes the space of Cesáro double ideal null sequences. Those notions are given as follows:

Definition 10. $C_{n m}^{I}=\left\{x=\left(x_{n m}\right) \in 2 \omega:\{(n, m) \in N \times N: I-\right.$ $\left.\lim _{k j \rightarrow \infty} \frac{1}{k j} \sum_{n m=1}^{k j}\left\|x_{n m}-L\right\|=0\right\}$, for some $\left.L \in C\right\} \in I$.

Definition 11. $C_{0 n m}^{I}=\left\{x=\left(x_{n m}\right) \in 2 \omega:\{(n, m) \in N \times N: I-\right.$ $\left.\left.\lim _{k j \rightarrow \infty} \frac{1}{k j} \sum_{n m=1}^{k j}\left\|x_{n m}\right\|=0\right\}\right\} \in I$.

Theorem 12. The sequences spaces $C_{n m}^{I}$ and $C_{0 n m}^{I}$ are linear.

Proof. Let $x=\left(x_{n m}\right)$ and $y=\left(y_{n m}\right)$, where $x, y \in C_{n m}^{I}$. Then, we have that

$$
\begin{aligned}
& I-\lim _{k j \rightarrow \infty} \frac{1}{k j} \sum_{n m=1}^{k j}\left\|x_{n m}-L\right\|=0 \text { for some } L \in C, \\
& I-\lim _{k j \rightarrow \infty} \frac{1}{k j} \sum_{n m=1}^{k j}\left\|y_{n m}-L_{0}\right\|=0 \text { for some } L_{0} \in C .
\end{aligned}
$$


Now, let

$$
\begin{aligned}
& \mathcal{V}_{1}=\left\{(n, m) \in N \times N: \frac{1}{k j} \sum_{n m=1}^{k j}\left\|x_{n m}-L\right\|\right\}, \\
& \mathcal{V}_{2}=\left\{(n, m) \in N \times N: \frac{1}{k j} \sum_{n m=1}^{k j}\left\|y_{n m}-L_{0}\right\|\right\} .
\end{aligned}
$$

Now, let $\gamma$ and $\phi$ be any two scalars. Taking into account the properties of norm, we have that

$$
\begin{gathered}
\lim _{k j \rightarrow \infty} \frac{1}{k j} \sum_{n m=1}^{k j}\left\|\left(\gamma x_{n m}+\phi y_{n m}\right)-\left(\gamma L+\phi L_{0}\right)\right\| \\
\leq \lim _{k j \rightarrow \infty} \frac{1}{k j}|\gamma|\left\|x_{n m}-L\right\|+\lim _{k j \rightarrow \infty} \frac{1}{k j}|\phi|\left\|y_{n m}-L_{0}\right\| .
\end{gathered}
$$

Thus, from (1) and (2), we have that for every $\epsilon>0$,

$$
\begin{gathered}
\left\{(n, m) \in N \times N: \lim _{k j \rightarrow \infty} \frac{1}{k j} \sum_{n m=1}^{k j}\left\|\left(\gamma x_{n m}+\phi y_{n m}\right)-\left(\gamma L+\phi L_{0}\right)\right\|\right. \\
\geq \epsilon\} \subset \mathcal{V}_{1} \cup \mathcal{V}_{2} .
\end{gathered}
$$

Therefore, $\left(\gamma x_{n m}+\phi y_{n m}\right) \in C_{n m}^{I}$ for all scalars $\gamma, \phi$ and $\left(x_{n m}\right),\left(y_{n m}\right) \in C_{n m}^{I}$. In consequence, this implies that $C_{n m}^{I}$ is a linear space. $C_{n m}^{I}$.

The proof of $C_{0 \mathrm{~nm}}^{I}$ is a linear space is proved in the same manner of the

Proposition 13. Let $x=\left(x_{n m}\right) \in \omega$ be any double sequence, then $C_{0 n m}^{I} \subset C_{n m}^{I}$.

Proof. The proof is followed by the Definitions 10 and 11 .

Theorem 14. The space $C_{0 n m}^{I}$ is solid.

Proof. Let $\left(x_{n m}\right) \in C_{0 n m}^{I}$ be any element. Then, we have that

$$
\left\{(n, m) \in N \times N: I-\lim _{k j \rightarrow \infty} \frac{1}{k j} \sum_{n m=1}^{k j}\left\|x_{n m}\right\|=0\right\} .
$$


Now, let $\left(\gamma_{n m}\right)$ be a double sequence of scalars such that $\left|\gamma_{n m}\right| \leq 1$, for all $n m \in N \times N$. So, we gets that

$$
\frac{1}{k j} \sum_{n m=1}^{k}\left|\gamma_{n m}\right| \leq 1
$$

Then, from the above inequality, we have that

$$
\begin{gathered}
\frac{1}{k j} \sum_{n m=1}^{k j}\left\|\gamma_{n m} x_{n m}\right\|=\frac{1}{k j} \sum_{n m=1}^{k j}\left|\gamma_{n m}\right|\left\|x_{n m}\right\| \\
=\frac{1}{k j} \sum_{n m=1}^{k j}\left|\gamma_{n m}\right| \frac{1}{k j} \sum_{n m=1}^{k j}\left\|x_{n m}\right\| \leq \frac{1}{k j} \sum_{n m=1}^{j k}\left\|x_{n m}\right\|
\end{gathered}
$$

for all $(n, m) \in N \times N$. Therefore, the space $C_{0 n m}^{I}$ is solid.

Theorem 15. A double sequence $x=\left(x_{n m}\right) \in C_{n m}^{I}$ is double $I$-convergent if and only if for every $\epsilon>0$, there exists $t=t(\epsilon) \in N \times N$ such that

$$
\left\{(n, m) \in N \times N: \frac{1}{k j} \sum_{n m}^{k j}\left\|x_{n m}-x_{t}\right\|<\epsilon\right\} \in F(I) .
$$

Proof. We begin proof $\Rightarrow$ :

Consider $x=\left(x_{n m}\right) \in C_{n m}^{I}$. Then, $I$ - $\lim _{k j \rightarrow \infty} \frac{1}{k j} \sum_{n m}^{k j}\left\|x_{n m}-L\right\|=0$. Thus, for all $\epsilon>0$ the set

$$
C_{n m}^{\epsilon}=\left\{(n, m) \in N \times N: \frac{1}{k j} \sum_{n m}^{k j}\left\|x_{n m}-L\right\|<\frac{\epsilon}{2}\right\} \in F(I) .
$$

Fix a $t(\epsilon) \in C_{n m}^{\epsilon}$, then we obtain

$$
\frac{1}{k j} \sum_{n m}^{k j}\left\|x_{n m}-x_{t}\right\| \leq \frac{1}{k j} \sum_{n m}^{k j}\left\|x_{n m}-L\right\|+\frac{1}{k j} \sum_{n m}^{k j}\left\|x_{t}-L\right\|<\frac{\epsilon}{2}+\frac{\epsilon}{2}=\epsilon
$$

which holds for all $(n, m) \in C_{n m}^{\epsilon}$. Therefore,

$$
\left\{(n, m) \in N \times N: \frac{1}{k j} \sum_{n m}^{k j}\left\|x_{n m}-x_{t}\right\|<\epsilon\right\} \in F(I) .
$$


Now, we proof $\Leftarrow$ :

Consider that for all $\epsilon>0$, the set

$$
\left\{(n, m) \in N \times N: \frac{1}{k j} \sum_{n m}^{k j}\left\|x_{n m}-x_{t}\right\|<\epsilon\right\} \in F(I) .
$$

Then, for every $\epsilon>0$, we have that

$$
\begin{gathered}
M_{n m}^{\epsilon}=\left\{(n, m) \in N \times N: \frac{1}{k j} \sum_{n m}^{k j}\left\|x_{n m}\right\|\right. \\
\left.\in\left[\frac{1}{k j} \sum_{n m}^{k j}\left\|x_{t}\right\|-\epsilon, \frac{1}{k j} \sum_{n m}^{k j}\left\|x_{t}\right\|+\epsilon\right]\right\} \in F(I) .
\end{gathered}
$$

We will denote $W_{n m}^{\epsilon}=\left[\frac{1}{k j} \sum_{n m}^{k j}\left\|x_{t}\right\|-\epsilon, \frac{1}{k j} \sum_{n m}^{k j}\left\|x_{t}\right\|+\epsilon\right]$.

For fixed $\epsilon>0$, we have that $M_{n m}^{\epsilon} \in F(I)$, as well as, $M_{n m}^{\epsilon / 2} \in F(I)$. Therefore, $M_{n m}^{\epsilon} \cap M_{n m}^{\epsilon / 2} \in F(I)$. This implies that $M_{n m}^{\epsilon} \cap M_{n m}^{\epsilon / 2} \neq \emptyset$. Thus,

$$
\left\{(n, m) \in N \times N: \frac{1}{k j} \sum_{n m}^{k j}\left\|x_{n m}\right\| \in W_{n m}\right\} \in F(I) .
$$

For this, we have $\operatorname{diam}\left(W_{n m}\right) \leq \operatorname{diam}\left(W_{n m}^{\epsilon}\right)$, where $\operatorname{diam}\left(W_{n m}\right)$ denotes the length of the interval of $W_{n m}$. In this way, by induction, we have the sequence of closed intervals $W_{n m}^{\epsilon}=U_{n m}^{0} \supseteq U_{n m}^{1} \supseteq \ldots \supseteq U_{n m}^{5} \supseteq \ldots$. With the property that $U_{n m}^{i} \leq 1 / 2 \operatorname{diam}\left(U_{n m}^{i-1}\right)$, for $i=1,2,3, \ldots$ and

$$
\left\{(n, m) \in N \times N: \frac{1}{k j} \sum_{n m}^{k j}\left\|x_{n m}\right\| \in U_{n m}^{i}\right\} \in F(I),
$$

for $i=1,2,3, \ldots$. Then, there exists a $L \in \cap U_{n m}^{i}$ such that

$$
L=I-\lim _{k j \rightarrow \infty} 1 / k j \sum_{n m}^{k j}\left\|x_{n m}\right\|
$$

This proves that $x=\left(x_{n m}\right) \in C_{n m}^{I}$ is double $I$-convergent.

Theorem 16. Let $x=\left(x_{n m}\right)$ and $y=\left(y_{n m}\right)$ be any two double sequences such that $T(x \cdot y)=T(x) T(y)$. Then, the space $C_{n m}^{I}$ and $C_{0 n m}^{I}$ are sequence algebra. 
Proof. Let $x=\left(x_{n m}\right)$ and $y=\left(y_{n m}\right)$ be any two elements of $C_{n m}^{I}$ with $T(x \cdot y)=T(x) T(y)$. Now, for every $\epsilon>0$ choose $\lambda>0$ such that $\epsilon<\lambda$. Then, we have that

$$
\left\{(n, m) \in N \times N: \frac{1}{k j} \sum_{n m}^{k j}\left\|T\left(x_{n m}\right)-L_{i}\right\|<\frac{\epsilon}{2 \lambda}\right\} \in F(I)
$$

and

$$
\left\{(n, m) \in N \times N: \frac{1}{k j} \sum_{n m}^{k j}\left\|T\left(y_{n m}\right)-L_{p}\right\|<\frac{\epsilon}{2 L_{i}}\right\} \in F(I) .
$$

Taking into account the above and the properties of norm, we have that

$$
\begin{gathered}
\frac{1}{k j} \sum_{n m}^{k j}\left\|T\left(x_{n m} y_{n m}\right)-L_{i} L_{p}\right\| \\
=\frac{1}{k j} \sum_{n m}^{k j}\left\|T\left(x_{n m}\right) T\left(y_{n m}\right)-L_{i} L_{p}\right\| \\
=\frac{1}{k j} \sum_{n m}^{k j}\left\|T\left(x_{n m}\right) T\left(y_{n m}\right)-L_{i} T\left(y_{n m}\right)+L_{i} T\left(y_{n m}\right)-L_{i} L_{p}\right\| \\
\leq \frac{1}{k j} \sum_{n m}^{k j}\left\|T\left(y_{n m}\right)\right\| \frac{1}{k j} \sum_{n m}^{k j}\left\|T\left(x_{n m}\right)-L_{i}\right\|+\left|L_{i}\right| \frac{1}{k j} \sum_{n m}^{k j}\left\|T\left(y_{n m}\right)-L_{p}\right\| \\
<\frac{\epsilon^{2}}{2 \alpha}+\left|L_{i}\right| \frac{\epsilon}{2\left|L_{i}\right|}<\epsilon .
\end{gathered}
$$

Hence, the set

$$
\left\{(n, m) \in N \times N: \frac{1}{k j} \sum_{n m}^{k j}\left\|T\left(x_{n m} y_{n m}\right)-L_{i} L_{p}\right\| \geq \epsilon\right\} \in I .
$$

In consequence, $\left(x_{n m}\right)\left(y_{n m}\right) \in C_{n m}^{I}$. Therefore, $C_{n m}^{I}$ is a sequence algebra. The proof of $C_{0 n m}^{I}$ is a sequence algebra is proved in the same manner of the $C_{n m}^{I}$. 


\section{Conclusion}

In this paper, we defined and studied some properties on $C_{n m}^{I}$ and $C_{n m 0}^{I}$ spaces, as well as, the closed relation between them. On the other hand, those concepts let the researchers extend and prove new notions on these spaces taking into account double sequences in ideal spaces. Otherwise, during the developing of this paper, we had been thinking about the following question: Is the space $C_{n m}^{I}$ solid ? It would be interesting to show or to prove this result which is an open problem.

\section{References}

[1] M. Faisal, Some results on strongly Cesáro ideal convergent sequence spaces, Journal of Mathematics, 2020 (2020), 1-4.

[2] G. Hardy and J. Littlewood, Sur la série de fourier dúne fonction a carré sommable, Comptes rendus de l'Académie des Sciences, 156 (1913), 13071309.

[3] V. Khan, H. Fatima, S. Addullah and K. Alshlool, On paranorm $B V_{\sigma}$ $I$-convergence double sequence spaces defined by an Orlicz function, Analysis, 37, No 3 (2017), 157-167.

[4] H. Nakano, Concave modulars, Journal of the Mathematical Society of Japan, 5, No 1 (1953), 29-49.

[5] T. Salat, B. C. Tripathy, and M. Ziman, On some properties of $I$ convergence, Tatra Mountains Mathematical Publications, 28 (2004), 279286.

[6] H. Sengul, Some Cesaro-type summability spaces defined by a modulus function of order $(\alpha, \beta)$, Communications Faculty of Sciences University of Ankara Series A1-Mathematics and Statistics, 66, No 2 (2017), 80-90.

[7] I. Maddox, Sequence spaces defined by a modulus, Mathematical Proceedings of the Cambridge Philosophical Society, 100, No 1 (1986), 161-166.

[8] M. Et and H. Sengul, Some cesaro-type summability spaces of order $\alpha$ and lacunary statistical convergence of order $\alpha$, Filomat, 28, No 8 (2014), 1593-1602. 
[9] W. Ruckle, FK spaces in which the sequence of coordinate vectors is bounded, Canadian Journal of Mathematics, 25, No 5 (1973), 973-978.

[10] H. Fast, Sur la convergence statistique, Colloquium Mathematicum, 2, No 3-4 (1951), 241-244.

[11] H. Steinhaus, Sur la convergence ordinaire et la convergence asymptotique, Colloquium Mathematicum, 2, No 1 (1951), 73-74.

[12] J. Fridy, On statistical convergence, Analysis, 5 (1985), 301-313.

[13] J. Fridy, Statistical limit points, Proceedings of the American Mathematical Society, 118, No 4 (1993), 1187.

[14] P. Kostyrko, T. Wilczynski, and W. Wilczynski, I-conver-gence, Real Analysis Exchange, 26, No 2 (2000), 669-686. 
